\title{
Experimental evaluation of amaranth food products in terms of functional food ingredients
}

\author{
A.A. Stakhurlova*, N.M. Derkanosova, A.V. Aristov, I.N. Ponomareva, and A.A. Sutolkin
}

Voronezh State Agrarian University, 394087 Voronezh, Russia

\begin{abstract}
Diet correction according to the nutrition enrichment with physiologically valuable nutrients becomes one of the most important issues. Its solution is possible by inclusion in the formulation of food products of raw materials that are characterized by an increased content of deficient substances, primarily protein, dietary fiber, minerals and vitamins. The amaranth meets the criteria above. The use of amaranth processing products in food technologies makes possible to obtain specialized and functional products. In this case, better to conduct experimental confirmation of the expected effect. Studies have been conducted to evaluate the effect of grain and extrudate of whole grain of Universal Amaranth on laboratory animals. Mature male rat's stomach, liver, and kidneys served as a study material. The results of histological studies showed an improvement in the digestive system of animals and their general condition when grain and extrude Universal Amaranth was included in the diet.
\end{abstract}

\section{Introduction}

The consumer market for food products is an essential part of the modern economy [1]. In turn, the quality and safety of food products is a factor in ensuring public health [2]. Proper nutrition determines the normal growth and development of the body, performs preventive functions, respectively, increasing its performance and adaptation to the environment [2-4]. Issues of a healthy nutrition requires innovative approaches, both to the composition of the recipe ingredients of food products and the formation of their consumer properties. Amount of amaranth is referred to quite often mentioned raw materials with a rich nutrient composition $[5,6]$.

Amaranth grain is high in protein, dietary fiber, minerals such as calcium and iron. It contains squalene, known for its antioxidant properties [7,8]. It is also possible to use amaranth leaf mass.

However, an analysis of the nutrient composition shows the advantages of the grain mass, especially if the task is set of its complex application. Promising products for processing amaranth grain include amaranth extrudate, obtained by grinding extruded amaranth grain mass [9].

At the same time, numerous studies in the field of obtaining products of amaranth processing and their application in food technologies require confirmation of functional and / or enriching properties, which is possible by evaluating the extrudate IN VIVO. The research is devoted to the study of this aspect of the characteristics of amaranth processing products (amaranth grain and amaranth grain extrudate).

\section{Objects and research methods}

A preliminary screening of the chemical composition of the grain screened amaranth varieties of the Voronezh breeding made it possible to distinguish Universal amaranth as an object of study. It is characterized by a high content of protein, lipids, minerals, including phosphorus, calcium, iron, as well as trace elements such as zinc and manganese. Extruded amaranth grain was obtained from whole semi-defatted grains of amaranth of the Universal variety on a laboratory universal smallsized extruder (EUS-1) at a temperature of $110-120^{\circ} \mathrm{C}$. Then crushed to granulometry no more than 125 microns.

The versatile amaranth extrudate is a loose powdery semi-finished product, light cream in color with a nutty flavor and a faint bitter taste characteristic of amaranth. It contains $4.76 \%$ moisture, $27.51 \%$ protein, $4.53 \%$ fat, $3.20 \%$ fiber.

The experiments to determine the effect of amaranth extrudate on metabolic processes in the body were performed on healthy sexually mature female Wistar rats weighing 210-240 g, which were kept in the vivarium. Animal welfare and experimental design were held following the international standards by the European Convention for the Protection of Vertebrate Animals (Strasbourg, 1986). White rats were in standard plastic cages of the company on a bed of fine wood shavings. The air temperature in the vivarium is $20-24{ }^{\circ} \mathrm{C}$, humidity is $50 \pm 20 \%$, the volume of air exchange (exhaust: supply) is $8: 10$, and the light mode (day: night) is 1: 1. Animals had constant access to water and food.

There were female rats of 9 individuals used in the experiment. Prior to the experiment, rats were fed oats

* Corresponding author: Stahurlova.n@mail.ru 
and barley for 2 weeks and watered in the public domain. The animals were kept: 1 cage 3 individuals (control), 2 cages -3 individuals, 3 cages -3 rats. The animals were divided into 3 groups, the abundance in 1 group was 3 rats (control), 3 rats in the second and 3 rats in the third group. The cage was cleaned 2 times a week. They fed 1 time a day in the morning. Animals of the first (control) group received $190 \mathrm{~g}$ of the main diet (oats and barley). Animals of the second group of $90 \mathrm{~g}$ of the main diet and $15 \mathrm{~g}$ of extrudate Universal, the third group of $90 \mathrm{~g}$ of the main diet and $15 \mathrm{~g}$ of grain Universal.

After 12 days, the animals of the control and experimental groups were euthanized with an overdose of chloroform in compliance with the principles of humanity set forth in the European Community directives (86/609 / EEC) and the Helsinki Declaration, and in accordance with the requirements of the rules for working with experimental animals.

The study material was the stomach, liver, and kidneys of sexually mature male white rats. For histological examination, tissue samples were fixed in a $10 \%$ solution of neutral formalin. The fixed samples after washing in running water were subjected to dehydration by placing the test material in alcohols with increasing concentration and poured into paraffin according to the standard method. Histological cross sections 3-4 $\mu \mathrm{m}$ thick were stained with hematoxylineosin [10].

\section{Results and discussion}

The structure of the stomach was represented by the mucosa, submucosa, muscle and serous membrane during histological examination of the control group of rats,. There is a close contact of the mucous membrane with muscle. The surface of the gastric mucosa is covered with a single-layer prismatic epithelium over the entire surface, including the fossa. The mucous membrane had multiple folds and was lined with a single-layer columnar epithelium. Histological examination of the gastric mucosa revealed a moderate amount of mucus produced by columnar epithelium. The cells of the body and the bottom of the glands are colored basophilically more pronounced than the excretory ducts. The cells of the glands are placed in the form of continuous cords, tightly adjacent to each other. The central arrangement of nuclei in the cells was revealed. The nuclei had a spherical shape. Marked expansion of blood vessels and stasis. Destructive changes in the glandular epithelium of the gastric mucosa are diffusely observed. The glands are expanded.

Morphological examination of the liver of the control group revealed a radial arrangement of beams. Hepatocytes form cords tightly adjacent to each other. A focal arrangement of hepatocytes with foamy cytoplasm was revealed under the capsule. Individual hepatocytes merge into one enlightened field. In the central part of the lobule, an accumulation of liver cells with foamy cytoplasm was also detected. The hepatocyte cytoplasm ruptured diffusely and the cell contents entered the organ parenchyma. The nuclear apparatus of the liver cells is compacted, in some places karyolysis and karyorexis were observed.
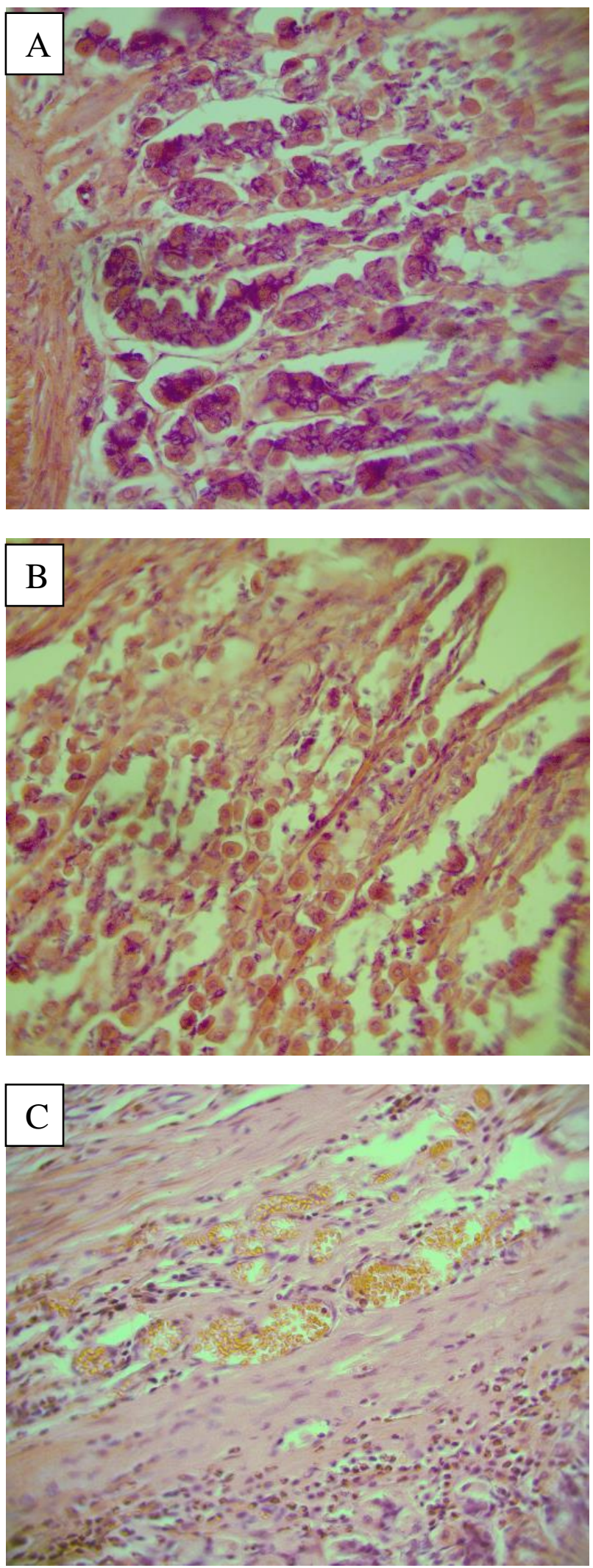

Fig. 1. The architectonics of the stomach of rats (control group). Hematoxylin-eosin stain. OK. $10 \times$ vol. $10(a, b, c)$ 

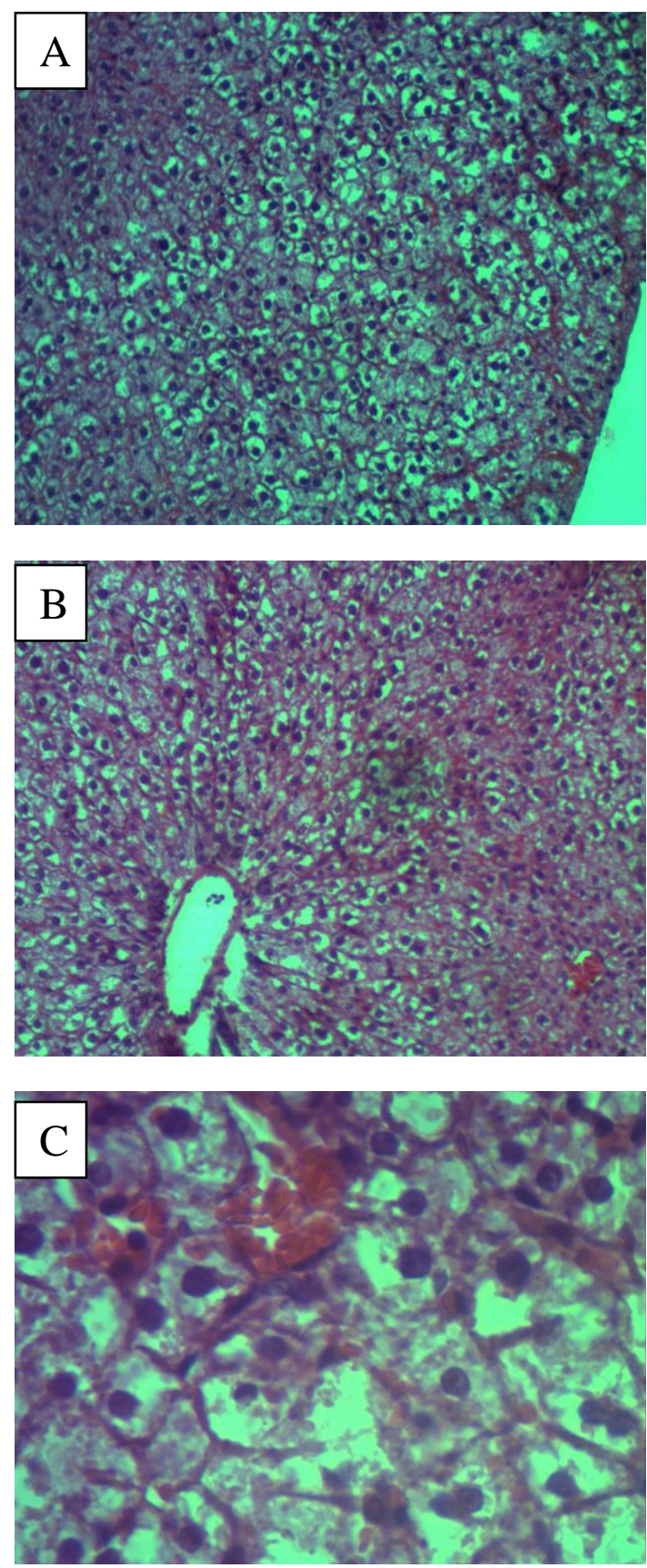

Fig. 2. Architectonics of rat liver (control group).

Hematoxylin-eosin stain. OK. $10 \times$ vol. $10(a, b), \times$ about 40 (c)

The objects observed: architectonics of the kidneys of the control group in white rats, plethora of the organ, separation into the cortical and brain layers. The renal corpuscles of regular round shape were in the cortical layer. The relative safety of glomerular tissues was observed. The convoluted tubules of both the cortical and the medulla are moderately expanded, the cellular composition of the lining epithelium is cylindrical, and the nuclei occupied, as a rule, a central location. In some places, the expansion of the microvasculature of the cortical layer of the kidneys and foci of hemorrhage in the kidney parenchyma were revealed. Protein masses were found in the gaps of the tubules. Slight expansion of the cavity of the Bowman-Shumlyansky capsule (Fig. 3).
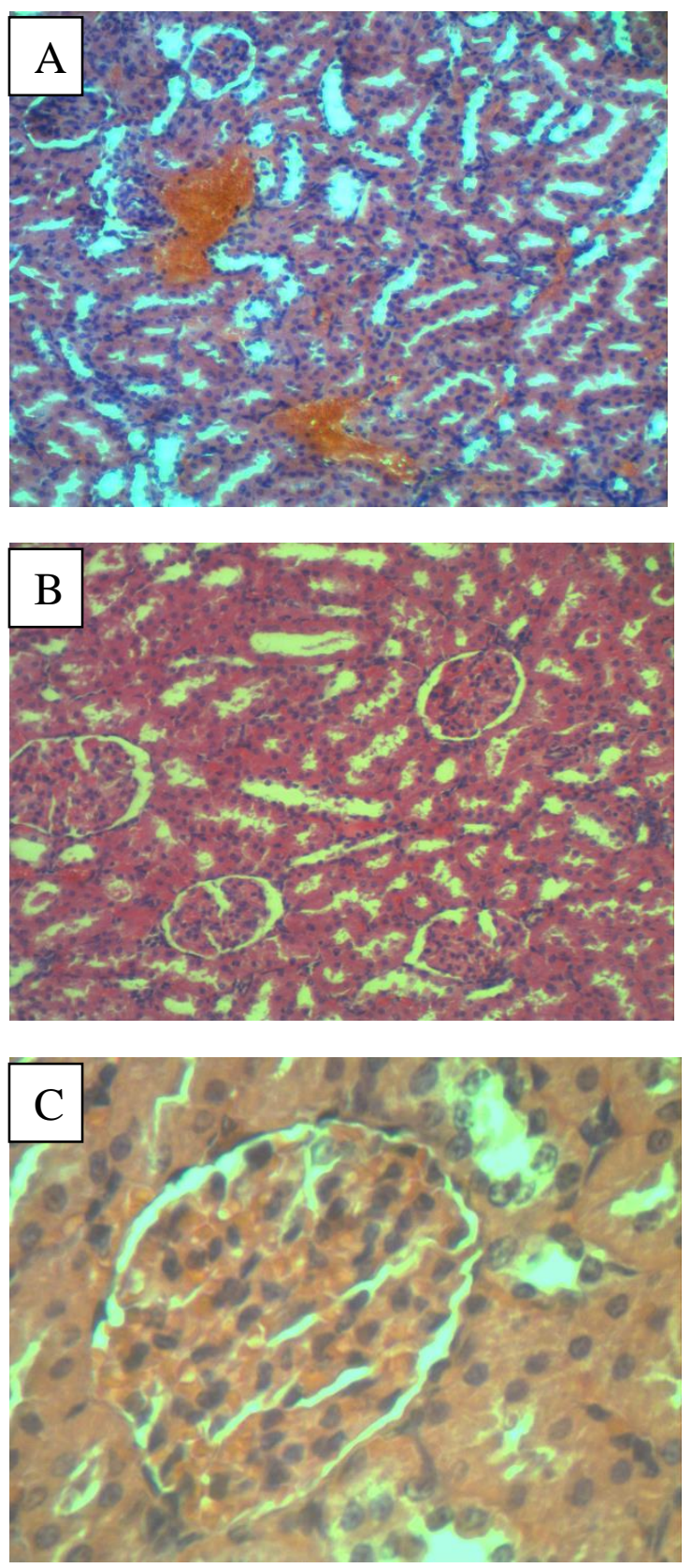

Fig. 3. Architectonics of the kidney of rats (control group). Hematoxylin-eosin staining. OK. $10 \times$ vol. $10(\mathrm{a}, \mathrm{b}), \times$ about 40 (c)

During histological examination of the stomach of rats of the second group, the architectonics is preserved, the organ is represented by four layers: the mucous membrane, submucosa, muscle layer, serous membrane. The mucous membrane is represented by a multilayer epithelium, in which fossae are clearly visible, from 3 to 6 layers of flat epithelial cells with a central location of the nucleus are clearly visible. The border between the multilayered epithelium and the glandular part is clearly visible, the cells of the glandular epithelium contained a moderate amount of secretion, without destructive changes. The mucous membrane of the glandular part is represented by a single-layer cylindrical epithelium lining the gastric fossa, at the base of which the glands of the stomach open. The submucous base does not contain glands, but a normal structure and thickness. It contains 
the vascular plexus and the neural submucous plexus of Meissner. The muscular membrane is well developed and consists of three layers. Outside, the stomach is covered with a serous membrane, consisting of a singlelayered squamous epithelium and a layer of connective tissue (Fig. 4).
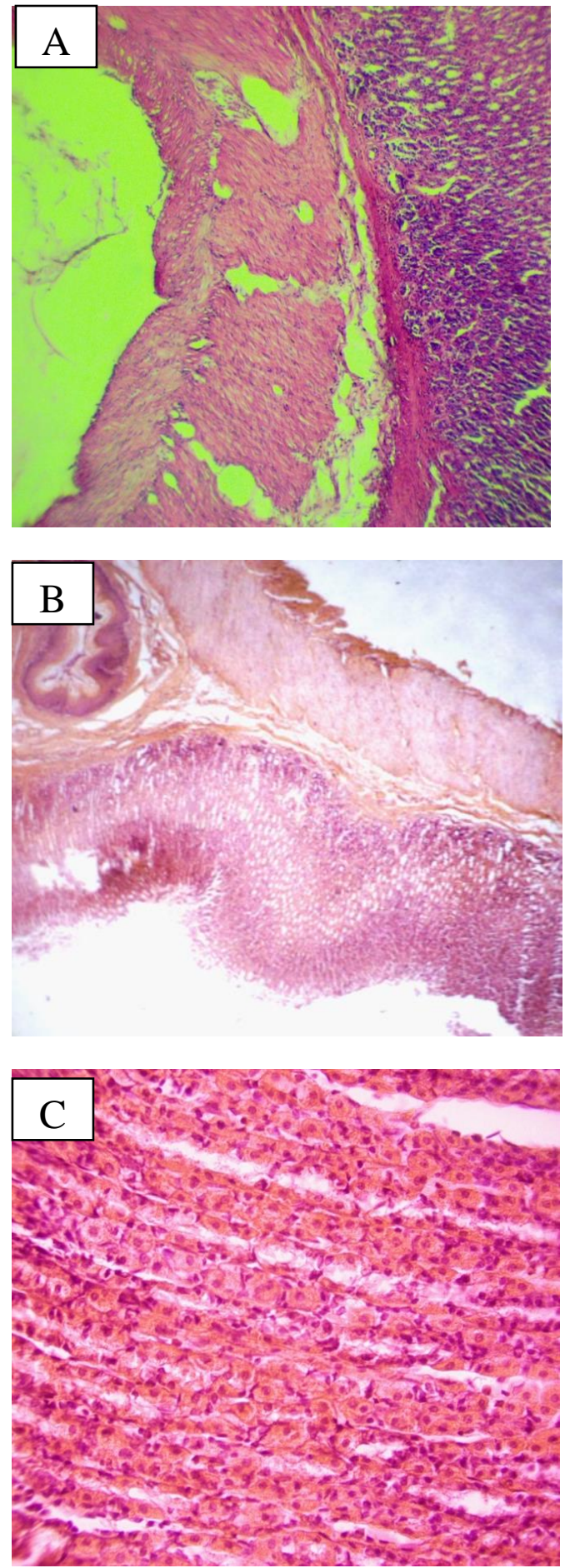

Fig. 4. Architectonics of the stomach of rats (second group). Hematoxylin-eosin stain. OK. $10 \times$ vol. $10(a, b) \times$ vol. $40(\mathrm{c})$

With a morphological study of the kidney, the structural organization of the organ is preserved. The kidney is represented by the cortical and cerebral layer, the boundaries between the layers are clearly distinguishable, the vessels of the cortical and cerebral layer were characterized by diffuse venous-capillary blood filling. The cortical substance is located under the capsule, consists of renal bodies. There are tubules of the nephron and connective tissue layers between them. The brain substance is located in the central part of the organ, consists of loops of nephrons, collecting tubes, papillary tubules and connective tissue layers between them. The renal corpuscles were characterized by a normal morphofunctional state: the renal glomerulus epithelium had no destructive changes, the Bowman-Shumlyansky capsule also had no pathologies. The renal pelvis contained moderate amounts of urine, the cells had a light cytoplasm and a centrally located nucleus without pathological changes (Fig. 5).
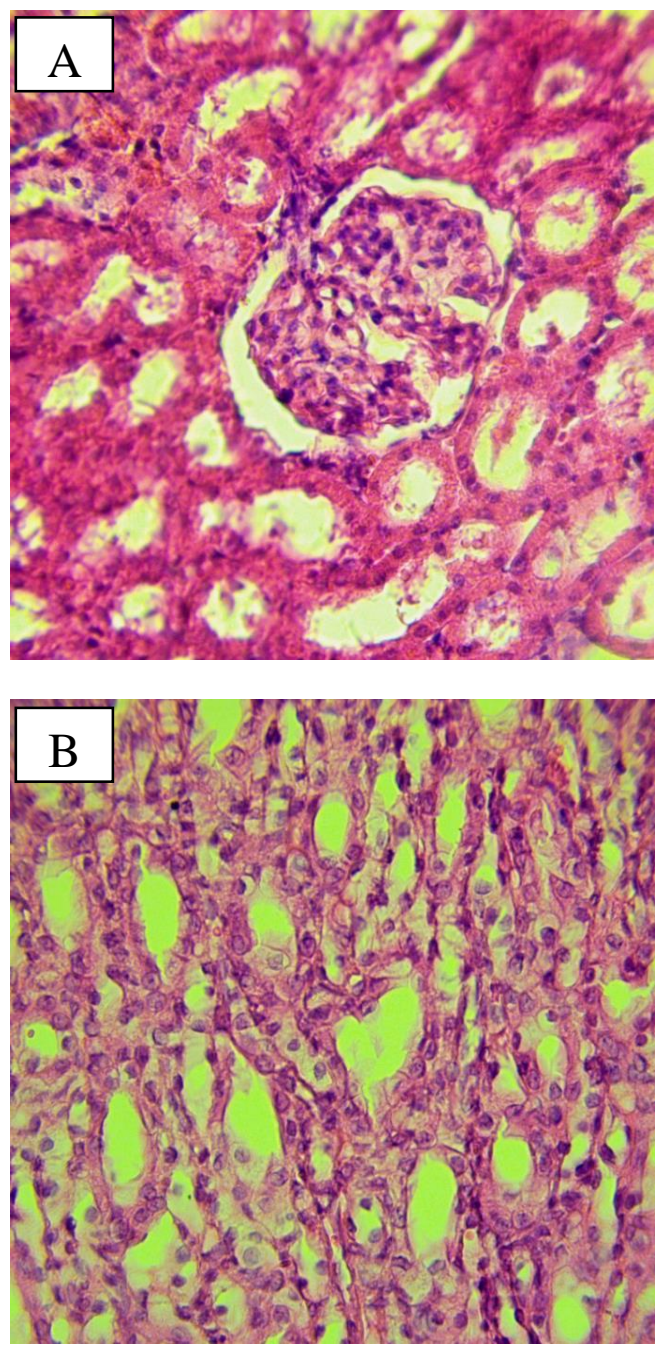

Fig. 5. Architectonics of the kidney of rats (second group). Hematoxylin-eosin stain. OK. $10 \times$ vol. 40 (a) $\times$ about 40 (b)

The structural organization of the organ is preserved during histological examination of the liver. The organ is represented by lobules having the correct hexagonal shape, lobules consist of radially located liver beams, there is a triad on the border: hepatic artery, vein and bile duct. Hepatocytes had a pale pink cytoplasm with centrally located nuclei. The nuclei were stained basophilically, in some the nucleoli were distinctly distinguished. The vessels of the organ were characterized by weak blood content (Fig. 6). 

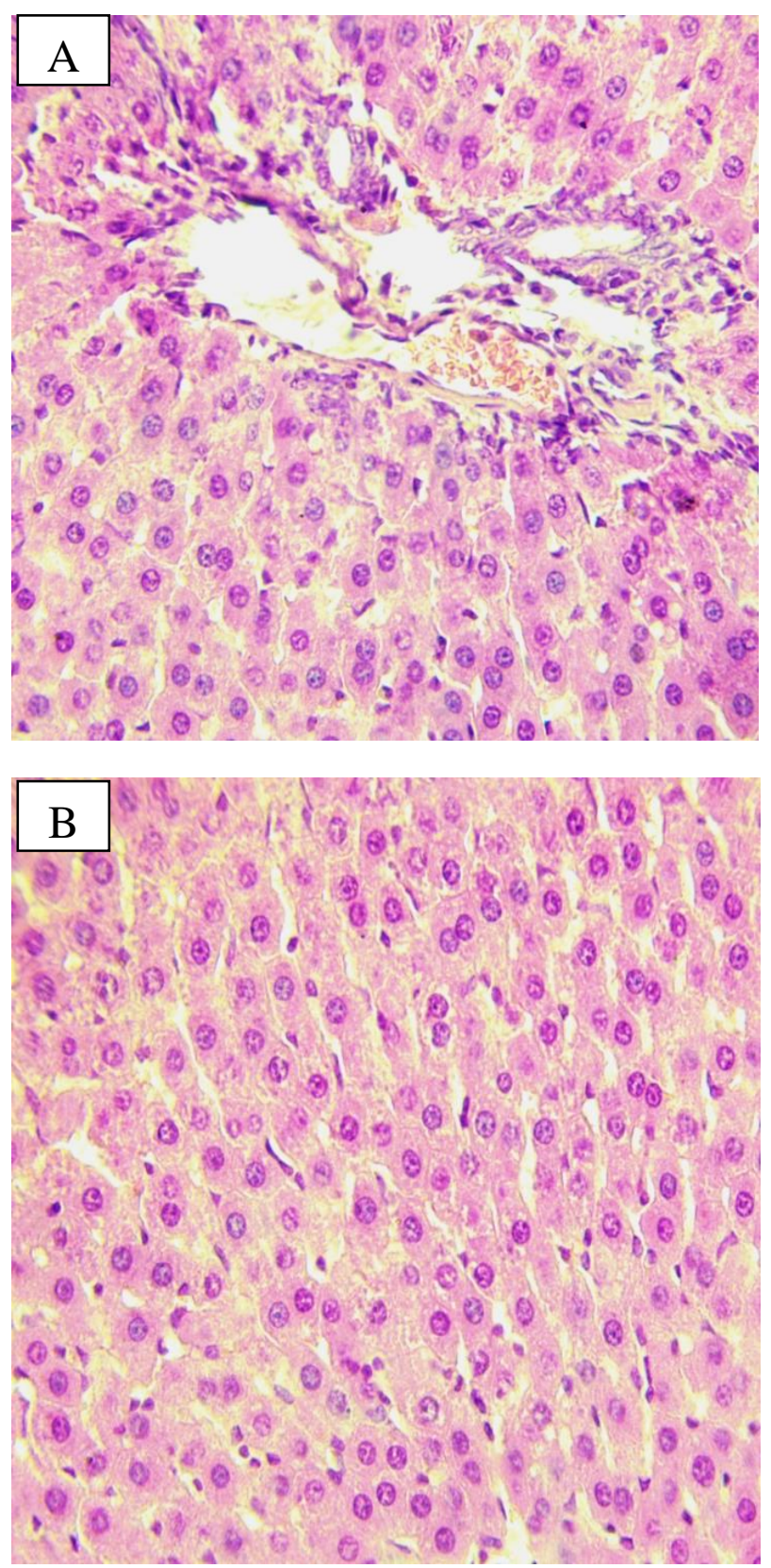

Fig. 6. The architectonics of rat liver (second group). Hematoxylin-eosin stain. OK. $10 \times$ vol. $40(a, b)$

During histological examination of the stomachs of rats of the third group, the architectonics was preserved. The organ is represented by 4 layers: the mucous membrane, the submucosa, the muscle layer and the serous membrane. The layer of mucous epithelium is represented by gastric fossa with a layer of epithelial cells, a single-layer highly prismatic epithelium and its own plate of the mucous membrane, in which several layers of glandular cells of the gastric epithelium were located. Between the mucosa and submucous membrane was a muscle plate. The submucosa was composed of blood vessels, which were characterized by diffuse haemorrhage. The muscular membrane included several layers of smooth muscle cells with elongated nuclei, and in some places, it was slightly thickened (Fig. 7).
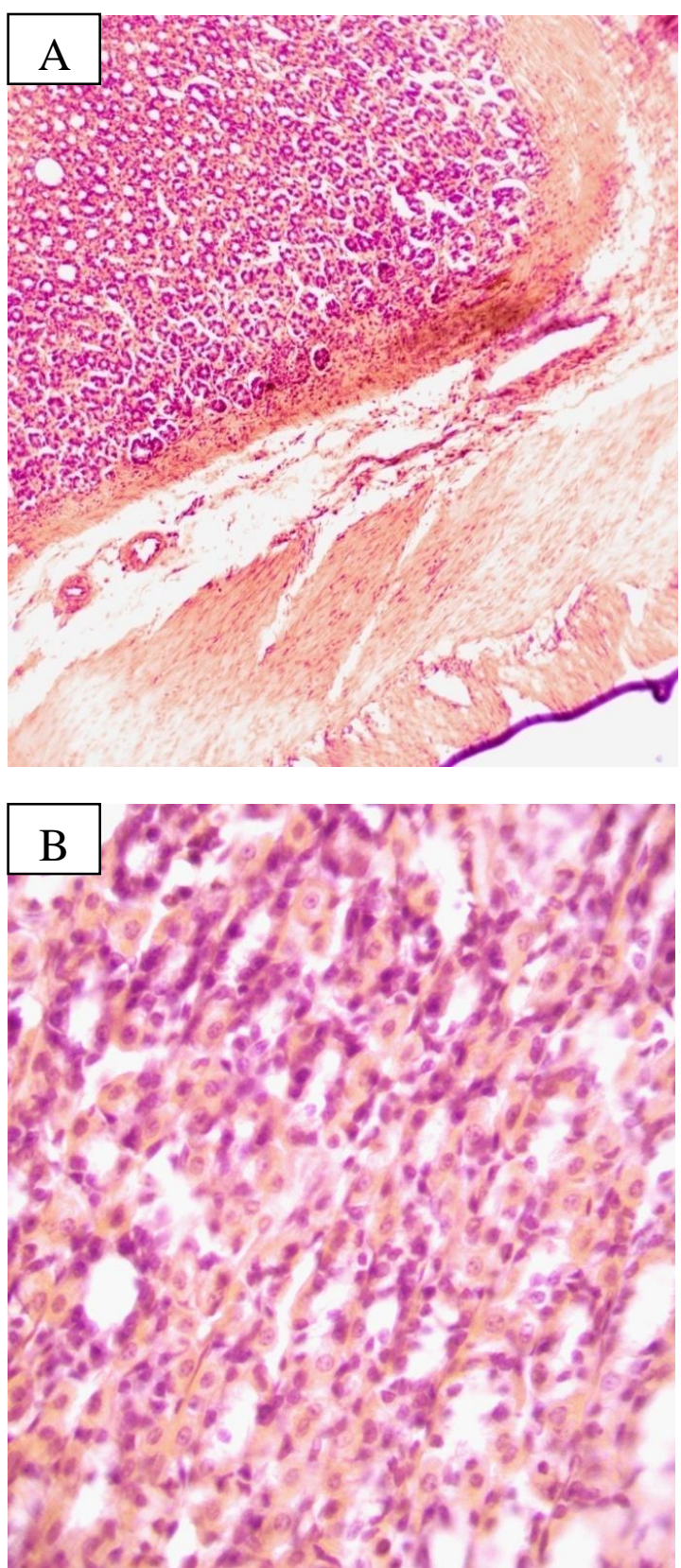

Fig. 7. The architectonics of the stomach of rats (third group). Hematoxylin-eosin stain. OK. $10 \times$ vol. 10 (a), $\times$ about 40 (b)

The cortical and cerebral layers are clearly distinguishable during histological examination of the kidney. The cortical layer is represented by distal and proximal convoluted tubules with diffusely located renal bodies. Nephrons had a normal morphofunctional state, the cavity of the Bowman-Schumlyanks capsule did not contain pathogenic exudates and had a normal state without thickenings. The brain substance lies in the central part of the organ, consists of loops of nephrons, tubules, papillary tubules and connective tissue layers between them. Blood vessels have diffused venouscapillary blood venation, without inflammatory cells in the lumen. The cells of the renal pelvis had a light cytoplasm of a pale pink hue; no crystals of urea or other pathogenic accumulations were found in the lumen of the output tubules (Fig. 8). 

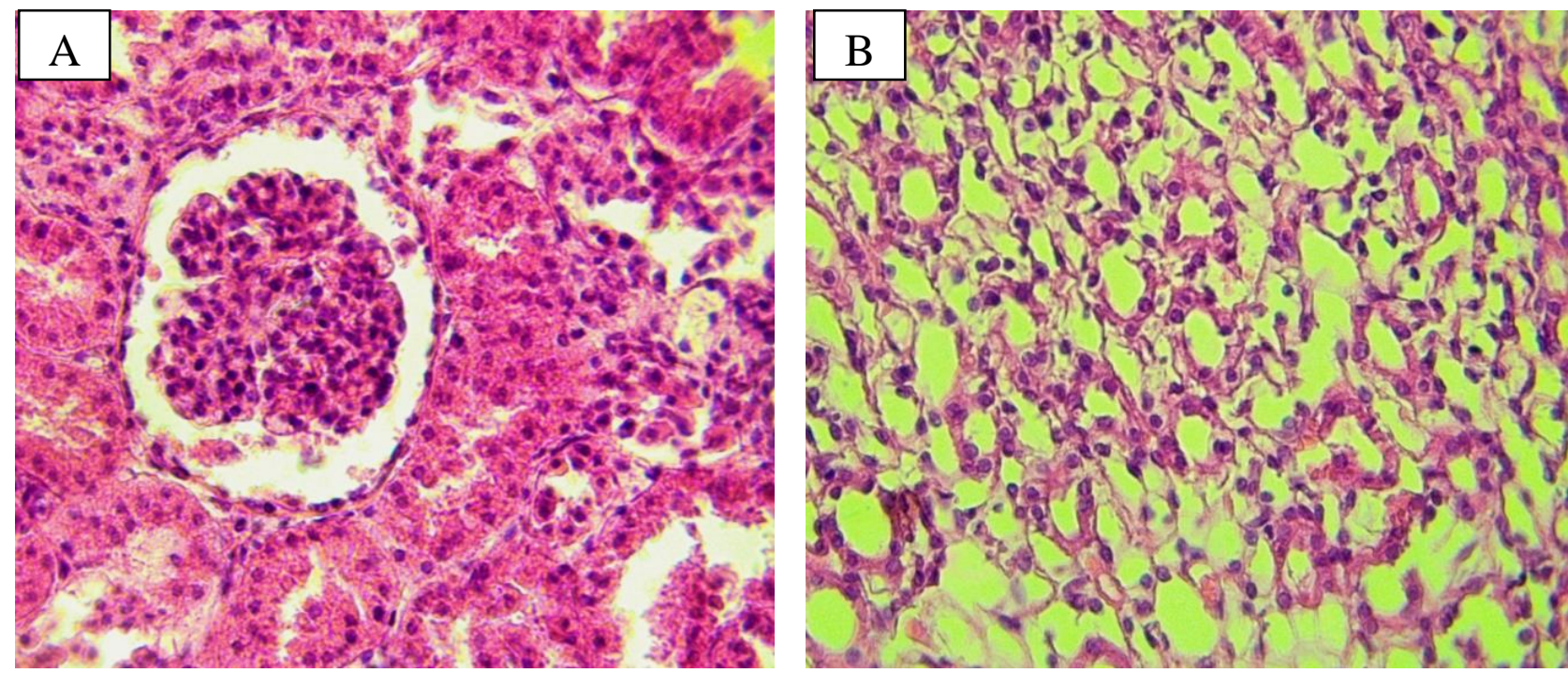

Fig. 8. Structural organization of the kidney of rats (third group). Hematoxylin-eosin staining. OK. $10 \times$ vol. $40(a, b)$
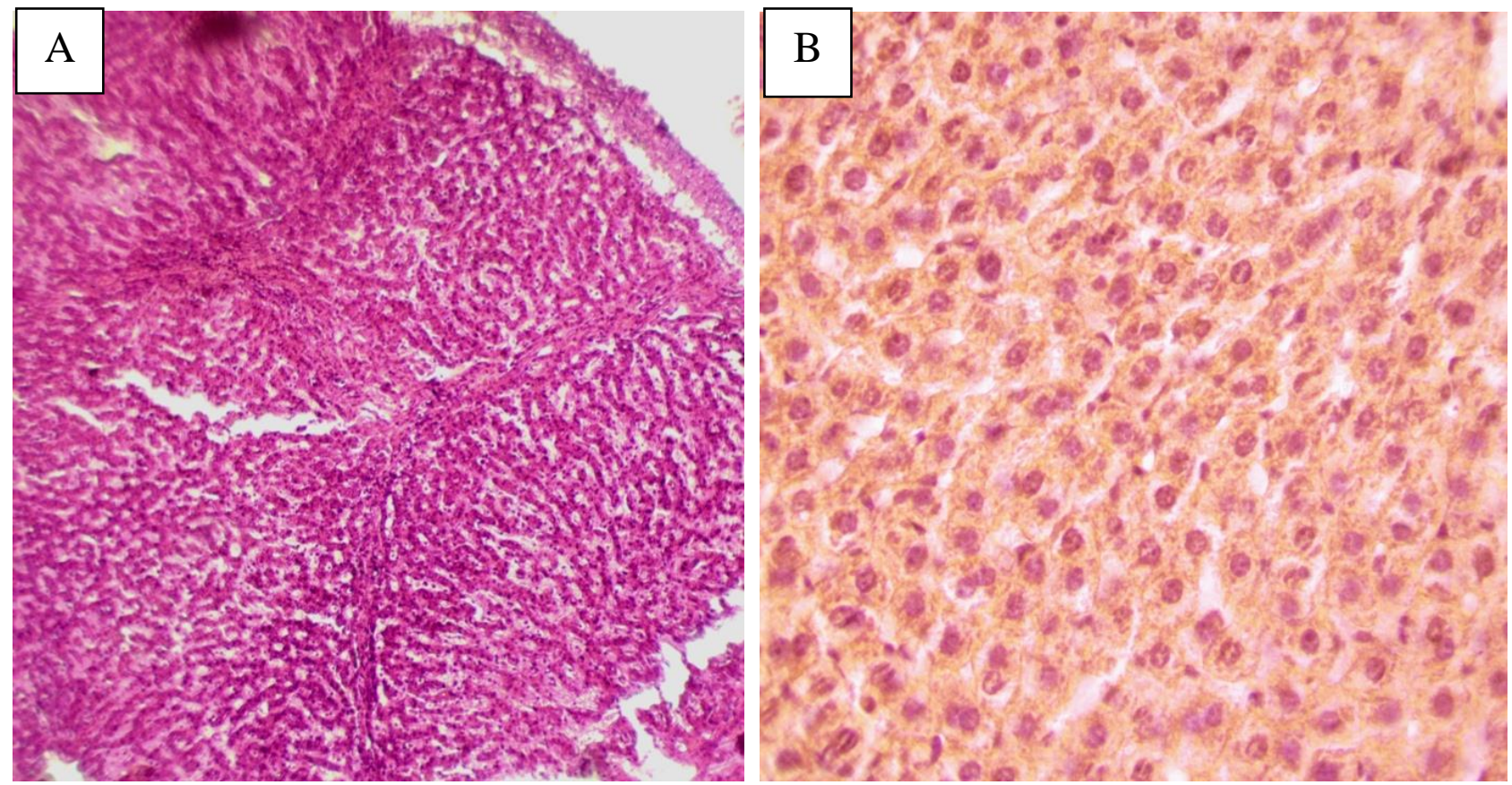

Fig. 9. Architectonics of rat liver (third group). Hematoxylin-eosin stain. OK. $10 \times$ vol. 10 (a), $\times$ about 40 (b)

The architectonics is preserved during histological examination of the liver: the beam-radial structure is clearly expressed, there is a triad of the liver at the borders of the beams (hepatic vein, artery, bile duct). The vessels had a weak blood supply, the walls was in a normal state, there was no parietal standing of red blood cells detected. Hepatocytes had a pale pink cytoplasm with a basophilically stained nucleus, the nucleolus of which could be clearly distinguished (Fig. 9).

\section{Conclusion}

Thus, histological studies of three animal groups revealed dystrophic processes in the liver in the rat control group, which led to metabolic disorders. In the second and third experimental groups, the morphological state of the digestive organs of rats indicates an improvement in the digestive system of animals and their general condition when switching to the extrudate and amaranth grain of the Universal Amaranth variety. According to the research the introduction of Universal Amaranth into the rat's diet provides protection and restorative properties of liver, and also regulates proliferative processes in organs and tissues, increases the enzymes activity. The above described results are also confirmed by biochemical studies of animal blood.

\section{References}

1. On approval of the Strategy for improving the quality of food products in the Russian Federation until 2030. Decree of the Government of the Russian Federation of 29 June 2016 no 1364-r. Retrieved from: Consultant-Plus system.

2. V.I. Pokrovsky, G.A. Romanenko, V.A. Knyazhev, N.F. Gerasimenko, G.G. Onishchenko, 
V.A. Tutelyan, V.M. Poznyakovsky, Health Food Policy: Federal and Regional Levels (Novosibirsk, 2002)

3. I.A. Rogov, E.N. Oreshkin, V.N. Sergeev, Medical and technological aspects of the development and production of functional food products Food Industry 1, 13-15 (2017)

4. M.S. Gins, V.F. Pivovarov, V.K. Gins, P.F. Kononkov, N.M. Derkanosova, Scientific support of innovative technologies when creating functional products based on vegetables, Vegetables of Russia, 1(22), 4-9 (2014)

5. I.A. Nikitin, The use of amaranth flour and modified compositions based on it in bread technology, $\mathrm{PhD}$ dissertation (Voronezh, 2005)

6. I.M. Zharkova, L.A. Miroshnichenko, A.A. Zvyagin, I.A. Bavykina, Amaranth flour: characteristic, comparative analysis, application possibilities, Nutrition issues, 83(1), 67-73 (2014)
7. L.I. Saratov, L.I. Saratovsky, A.L. Saratovsky, Grain and feed amaranth (Voronezh State Agrarian University, Voronezh, 2014)

8. M. Gins, V. Gins, V. Pivovarov, K.Kh. Torres Migno, P. Kononkov, Functional food products from amaranth seeds and leaves (VNIISSOK, Moscow, 2015)

9. N.M. Derkanosova, A.A. Stakhurlova, I.N. Ponomareva, O.A. Vasilenko, V.D. Lomova, M.V. Kopylov, Amaranth extrudate as an enriching ingredient in flour products, Bread products, 2, 32-34 (2018)

10. S.M. Suleymanov, A.V. Grebenshchikov, E.V. Mikhailov, I.S. Tolkachev et al., Methods of morphological studies, 2rd ed. (All-Russian Research Veterinary Institute of Pathology, Pharmacology and Therapy of the Russian Academy of Agricultural Sciences, Voronezh, 2007) 